\title{
Self-perceived evaluation of prenatal care: a hierarchical analysis by the users of Primary Health Care services in Brazil
}

\author{
Bruna Di Dea 1
}

iD https://orcid.org/0000-0002-7143-0492

Fernanda Andrade 2

iD https://orcid.org/0000-0002-9753-218X

Manoelito Ferreira Silva Junior 3

iD https://orcid.org/0000-0001-8837-5912

\footnotetext{
1,2 Departamento de Medicina. Universidade Estadual de Ponta Grossa. Ponta Grossa, PR, Brasil.

3 Departamento de Odontologia. Universidade Estadual de Ponta Grossa. Av. General Carlos Cavalcanti, 4748. Campus Uvaranas - Bloco M. Ponta Grossa, PR, Brasil. CEP: 84.030-900. E-mail: manoelito_fsjunior@hotmail.com
}

\begin{abstract}
Objective: to analyze the factors associated with positive self-perceived evaluation of prenatal care among users of Primary Health Care (PHC).

Methods: the analytical cross-sectional study was carried out with secondary data from the 3 rd Module of the 2nd cycle $(2013$ / 2014) of the External Evaluation of the Improvement of Access and Quality of Primary Care Program (PMAQ-AB - Portuguese acronym), collected from users in the Basic Health Units (BHU) that joined the PMAQ-AB in Brazil. The hierarchical analysis was performed using a theoretical model and logistic regression was performed between the self-perceived evaluation (positive - very good/good or negative regular/poor/very bad) and the sociodemographic characteristics, prenatal care and health service evaluation $(p<0.05)$.

Results: the sample consisted of 9,922 women and $81.7 \%$ rated care as very good/good. In the final model, positive evaluation was associated to women with incomplete higher education or over $(O R=1.05 ; C 195 \%=1.01-1.09 ; p=0.010)$, who underwent the VDRL exam $(O R=1.07 ; C 195 \%=1.01-1.14 ; p=0.020)$, consultation with the same professional $(O R=1.07$; $C 195 \%=1.02-1.12 ; p=0.010)$, received guidance on the gynecological preventive exam $(O R=1.05 ; C 195 \%=1.01-1.08 ; p=0.007)$, believed that guidance helped with pregnancy and child care $(O R=1.24 ; C 195 \%=1.05-1.46 ; p=0.012)$, evaluated the BHU structure as very good $/$ good $(O R=1.19 ; C 195 \%=: 1.14-1.24 ; p<0.001)$ and would not change BHU or team $(O R=1.62 ; C 195 \%=1.48-1.68 ; p<0.001)$.

Conclusion: the positive evaluation of prenatal care was associated with sociodemographic factors, prenatal care and health service evaluation.

Key words Prenatal care, Quality of health care, Self-perception
\end{abstract}

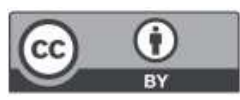




\section{Introduction}

Prenatal is the health service that pregnant women receive from conception to the start of labor. The main objective of the prenatal care is to assist women and promote birth of healthy children by guaranteeing maternal and neonatal wellbeing. ${ }^{1}$

It has been proven that good quality and humanized prenatal care reduces gestational risk and complications for both mother and baby, and to guarantee that, it is necessary to facilitate the access to users, with the assistance of qualified health professionals to develop both preventive and curing activities, along with an organized service network. 2,3

Aiming at improving the quality of the services offered to pregnant women, several health public policies have been implemented in Brazil.4 In 2000, Brazil became one of the signatory members of the United Nations Millennium Declaration, 5 which presented as one of its targets the maternal mortality reduction until 2020. In 2002, the Health Ministry started the implementation of the Prenatal and Birth Humanization Program (PHPN - Portuguese acronym), aiming to improve perinatal results, with emphasis on the importance of evaluating the quality of the service available to pregnant women. 6

In addition, from 2005 onwards, pregnant women acquired the legal right to be accompanied by a person of their choice throughout labor. In 2011, the federal government launched the Stork Network program, the maternal-infant care network whose aim is to give full attention to the health of pregnant and puerperal women, mothers and newborns, babies and toddlers up to 2 years old, with the purpose of ensuring humanized care and the birth of healthy children. ${ }^{4}$

After the implementation of prenatal care policies, measures to evaluate and monitor the routine of health services are needed to verify their effectiveness and/or revise the programs and actions carried out. Being aware of this need, Ministry of Health (MH) established the Program of Improvement of Access and Quality of Primary Care - (PMAQ-AB Portuguese acronym) as a way to guarantee a comparable standard of quality at the national, regional and local levels regarding the service provided by the Brazilian Health Unified System- (SUS - Portuguese acronym). ${ }^{7}$ Regarding the prenatal care assessment, most studies report the use of objective measures, and mainly through service offer.8-13 However, according to the Donabedian model, user's satisfaction is part of the process and therefore, is part of the external evaluation of the PMAQ-AB.7 Thus, studies evaluating health services and prenatal care should present parameters that include the users' perspective, 14-21 enabling a health planning with effective strategies for the prenatal care most inte-rested party, the users.

The national ${ }^{14-19}$ and international 20,21 literature is still scarce in relation to the evaluation or satisfaction from the standpoint of the women receiving prenatal care. It still presents a wide methodological diversity regarding both the quantitative $14-16,18,21$ and qualitative 17,19 approaches, either at the level of hospital care $15,16,20$ or at the Primary Health Care level14,17-19,21 with local coverage in municipalities and /or regions. ${ }^{14-21}$ In this sense, this study with a quantitative approach at the national level, aimed to analyze the factors associated to the self-perceived positive evaluation of prenatal care among users of the Primary Health Care service.

\section{Methods}

This is a quantitative, analytical and transversal study with secondary data from the Module III of the External Assessment (EA) of the 2nd Cycle of the Program of Improvement of Access and Quality of Primary Care - PMAQ-AB, which included 5,070 Brazilian municipalities (91\%) assisted by 30,424 Basic Care Teams (EAB - Portuguese acronym) $(90 \%)$ distributed throughout the five Brazilian regions.

The PMAQ-AB had a tripartite coordination by the Primary Care Department of the Ministry of Health - MH, Health Secretary National Council (CONASS - Portuguese acronym) and Municipal Health Secretary National Council - (CONASEMS - Portuguese acronym). The external assessment (EA) data collection was carried out using a multicentric approach with ten higher education institutions (HEI) as coordinating centers. This phase respected ethical aspects, and the data is in the public domain, being made available by the Health Ministry.

The adhesion to the PMAQ-AB was voluntary and offered to all Brazilian Primary Care Teams (EAB), assuming an initial process of partnership between the teams and municipal managers. Only the teams that formalized their adhesion by using the electronic form filled in by the municipal managers were evaluated.

The second cycle data collection occurred between November 2013 and July 2014, in loco, by external evaluators that used a tablet computer containing an application with the standardized and previously tested instrument, which comprised seven modules. The data was collected by interviewers that 
were previously trained using a field manual elaborated by the $\mathrm{DAB}$ and the participating HEI.

This study only included the data referring to the program external evaluation, regarding Module III, referring to the users' data. From each EAB evaluated, four users were selected. The module aimed to assess the users' satisfaction and perception of the health service access and use. In addition to genera questions, it presented sets of specific questions that were applied according to the users' profile (women, prenatal, child, hypertension or diabetes).

The intentional sample was obtained from the acceptance to participate in the research and the signature of the Free and Informed Consent Form (FICF). The participant's inclusion criterion was: users present in the basic health unit (BHU) at the moment of the external evaluation and that did not have an appointment with a doctor or nurse on the day of the interview. The exclusion criteria were: users' first time at the BHU, being under 18, and having not used the BHU services for a period over 12 months.

In this study, with the purpose of obtaining the sample, a filter was used in the data base, and the analysis included only women (code 2) that had already been pregnant (code 1), having had their last prenatal care at the BHU where the data collection was carried out (code 1) and that were currently under 50 years old.

The study outcome was the self-perceived evaluation of the prenatal care, which was categorized in a dichotomous way as: positive (very good / good) and negative (regular / bad / very bad). The independent variables were divided into five variable sets: sociodemographic factors, clinical and laboratory procedures, health service organization, health guidance and health service evaluation.

The sociodemographic factors observed were: age ( $<20$ years old; 20 to 34 years old; $>34$ years old); race (Caucasian or another - Afro-descendant, Asian, brown/interracial, indigenous, ignored); schooling (incomplete elementary school; incomplete high school; completed high school, incomplete higher education or more); job (yes or no); own income ( $<1$ minimum wage; $\geq 1$ minimum wage $)$; Bolsa Família - Federal Government Program of income transfer to poor families - beneficiary (receiving the benefit or not)

The variables related to clinical and laboratory procedures were: did you have your belly measured? (yes or not); did you have your blood pressure checked? (yes or not); did you have your mouth examined? (yes or not); did you have your breasts examined? (yes or not); did you have a gynecolo- gical examination? (yes or not); did you have a Papanicolaou test? (yes or not); did you have a urine test? (yes or not); were you tested for the human immunodeficiency virus (HIV)? (yes or not); did you have an examination for the Venereal Disease Research Laboratory - VDRL? (yes or not); did you get an ultrasound? (yes or not); did you get a glucose examination? (yes or not); did you get a tetanus vaccine? (yes or not); were you prescribed ferrous sulfate? (yes or no); were you prescribed vitamin B9? (yes or not); number of appointments $(\leq 5$ appointments and $\geq 6$ appointments), and need for emergency assistance during pregnancy (yes or no). The variables regarding the health service organization were: prenatal care provided by a physician (yes or not); prenatal care provided by a nurse (yes or not), appointments with the same professional (yes or not); leaving the BHU with the next appointment already scheduled (never/almost never /usually or always); informed about the maternity hospital where the delivery would occur (yes or not).

The health guidance variables were: instructions on diet and weight (yes or not); instructions on breastfeeding (yes or not); child care guidance (yes or not); preventive gynecological check-up guidance (yes or not); information about the pregnant woman support group (yes or not); took part in the pregnant woman support group (yes or not).

The variables related to the health service assessment were: the guidance received helped to learn about pregnancy and child care (definitely yes or yes, it sometimes helped/no); the pregnant woman support group helped (yes, no or did not participate); BHU structure evaluation (regular/bad/very bad or very good /good); and, desire to change team or BHU (yes or not).

The analysis of the data collected was assisted by the Statistical Package for the Social Science (SPSS) version 20.0 (IBM Corp., NY, United States). The descriptive analysis was presented using absolute (n) and relative frequencies (\%). A conceptual theoretical model 22 adapted for the study was used to direct the hierarchical analysis of the five independent variable sets in three blocks, namely: sociodemographic factors, prenatal care (clinical and laboratory procedures, health service organization and health guidance) and health service evaluation (Figure 1).

The statistical analysis was carried out using the chi-square association test and gross and adjusted logistic regression between the outcome (selfperceived assessment of the care received from the health team) and the independent variables (sociodemographic, prenatal care and health service evalua- 
Figure 1

Theoretical conceptual model of the self-perceived evaluation of health teams by female users of the Primary Health Care adapted to the study.22 Brazil, 2014.

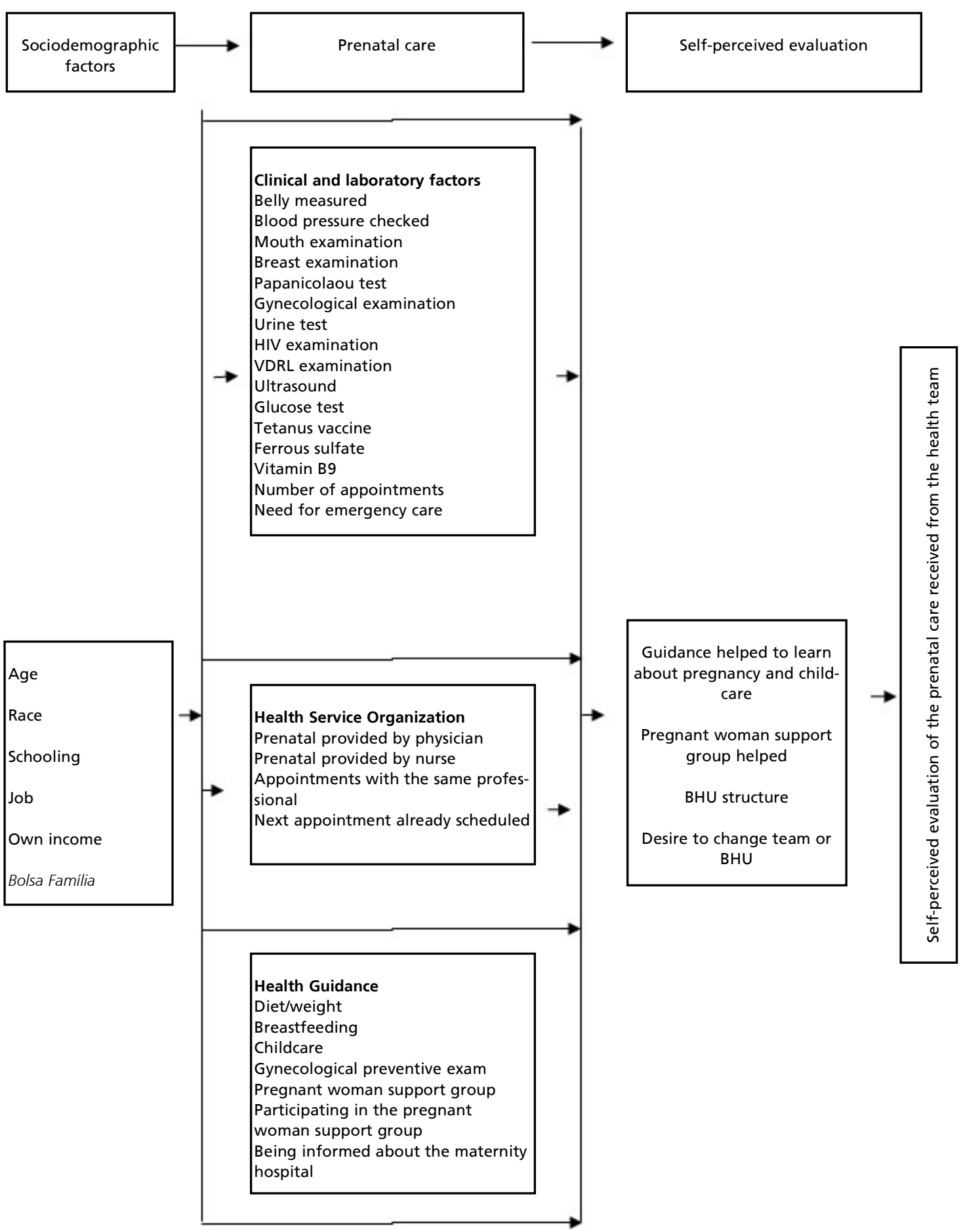


tion) with Odds Ratio (OR) definition and a 95\% confidence interval (CI95\%). For the model adjustment, the variables in the gross analysis with $p<0.20$ were selected, obtaining the final model assuming a $5 \%$ statistical significance level. The logistic regression reference category was the evaluation of the prenatal care as regular/bad/very bad.

\section{Results}

Out of the 114,615 users interviewed in the second cycle of the PMAQ-AB external evaluation, the interviews excluded belonged to 23,412 men, 29,945 individuals over 50 years old, 6,192 women that had never been pregnant, 45,133 women that did not have their prenatal care provided by the BHU evaluated, which resulted in 9,933 eligible women. This study sample included 9,922 women, and a sample loss of $0.1 \%(n=11)$ was recorded due to incomplete data.
Most women evaluated the primary health care team assistance as very good or good $(81.7 \%)$.

Most women were aged 20-34 years (75.3\%), had completed high school (33.0\%), were not Caucasian (71.1\%) and did not have a job (72.2\%), those who had a job declared an income over a minimum wage $(68.2 \%)$. In addition, most were not assisted by the Bolsa Família program1 (52.8\%). The positive evaluation of the assistance received from the health team was associated to all the sociodemographic variables of the users $(p<0.05)$ (Table 1).

Regarding prenatal care, the most used clinical and laboratory procedures were the urine test (97.6\%) and arterial pressure checking (98.8\%) with lower figures for the Papanicolaou test $(35.6 \%)$ and the gynecological examination $(41.4 \%)$. The variables related to the health service organization showed that most women left the BHU with the next appointment already scheduled $(86.6 \%)$, while few

Table 1

Sociodemographic factors associated to the self-perceived evaluation of the prenatal care received from the health team among women users of the Primary Health Care. Brazil, 2014.

\begin{tabular}{|c|c|c|c|c|c|c|c|}
\hline \multirow[t]{3}{*}{ Variables } & \multicolumn{6}{|c|}{ Self-perceived evaluation of the care received from the health team } & \multirow[t]{3}{*}{$p^{*}$} \\
\hline & \multicolumn{2}{|c|}{ Total } & \multicolumn{2}{|c|}{$\begin{array}{l}\text { Very good/ } \\
\text { Good }\end{array}$} & \multicolumn{2}{|c|}{$\begin{array}{c}\text { Regular/ Bad/ } \\
\text { Very bad }\end{array}$} & \\
\hline & $\mathrm{n}$ & $\%$ & $\mathrm{n}$ & $\%$ & $\mathrm{n}$ & $\%$ & \\
\hline \multicolumn{8}{|l|}{ Age (years) $(n=9922)$} \\
\hline $16-19$ & 1246 & 12.6 & 976 & 12.0 & 270 & 14.9 & $<0.001$ \\
\hline $20-34$ & 7476 & 75.3 & 6087 & 75.0 & 1389 & 76.9 & \\
\hline 35 or over & 1200 & 12.1 & 1052 & 13.0 & 148 & 8.2 & \\
\hline \multicolumn{8}{|l|}{ Race $(n=9785)$} \\
\hline Another & 6955 & 71.1 & 5600 & 70.0 & 1355 & 75.8 & $<0.001$ \\
\hline Caucasian & 2830 & 28.9 & 2397 & 30.0 & 433 & 24.2 & \\
\hline \multicolumn{8}{|l|}{ Schooling $(n=9919)$} \\
\hline Incomplete EE & 2859 & 28.8 & 2311 & 28.5 & 548 & 30.3 & $<0.001$ \\
\hline Incomplete HS & 3110 & 31.4 & 2477 & 30.5 & 633 & 35.0 & \\
\hline Completed HS & 3273 & 33.0 & 2725 & 33.6 & 548 & 30.3 & \\
\hline Incomplete HE or over & 677 & 6.8 & 599 & 7.4 & 78 & 4.3 & \\
\hline \multicolumn{8}{|l|}{ Job $(n=9922)$} \\
\hline No & 7457 & 75.2 & 6038 & 74.4 & 1419 & 78.5 & 0.001 \\
\hline Yes & 2465 & 24.8 & 2077 & 25.6 & 388 & 21.5 & \\
\hline \multicolumn{8}{|l|}{ Own income $(n=2170)$} \\
\hline$<1$ minimum wage & 691 & 31.8 & 556 & 30.4 & 135 & 39.7 & $<0.001$ \\
\hline 1 or over & 1469 & 68.2 & 1274 & 69.6 & 205 & 60.3 & \\
\hline \multicolumn{8}{|c|}{ Bolsa Família Program $(\mathrm{n}=9907)$} \\
\hline Not beneficiary & 5228 & 52.8 & 4347 & 53.6 & 881 & 48.8 & $<0.001$ \\
\hline Beneficiary & 4679 & 47.2 & 3756 & 46.4 & 923 & 51.2 & \\
\hline
\end{tabular}

$\mathrm{EE}=$ Elementary education; HS= High School; $\mathrm{HE}=$ Higher Education; ${ }^{*}$ Chi-square test $(p<0,05)$. 
women had their prenatal care provided by a professional that was not a physician or a nurse (1.2\%). As for health guidance, more instruction was received regarding breastfeeding $(90.8 \%)$ and diet $(88.0 \%)$, while the existence of a pregnant woman support group was mentioned on fewer occasions (54.8\%) Better self-perceived evaluation of prenatal care was observed among the women that had more clinical and laboratory procedures, reported better service organization and received more health guidance $(p<0.05)$ (Table 2).

When the variables related to the health service evaluation were analyzed, most participants pointed out that the guidance received during the prenatal care helped them to learn more about pregnancy and child care $(90.3 \%)$, while among those participating in the pregnant woman support group, most reported that the instruction received in the pregnant women's group helped them (98.5\%). A small number of users $(20.3 \%)$ stated that they would like to change the team or BHU. Greater perception of the prenatal care quality was observed among all the health service evaluation variables $(p<0.05)$ (Table 3 )

In the adjusted analysis, the best self-perceived evaluation of the care received from the health team was associated to sociodemographic factors such as schooling of the women with incomplete higher education or more $(\mathrm{OR}=1.05$; $\mathrm{CI} 95 \%=1.01-1.09$; $p=0.010$ ). In the prenatal care block, among clinical and laboratory procedures the associated factors were the VDRL examination $(\mathrm{OR}=1.07$; $\mathrm{C}$ 195\% $=1.01-1.14 ; p=0.020$ ), health service organization, among women that had been assisted by the same professional $(\mathrm{OR}=1.07$; CI95\% $=1.02-1.12$; $p=0.010$ ), and health guidance such as having received gynecological preventive examination instruction $(\mathrm{OR}=1.05 ; \mathrm{CI} 95 \%=1.01-1.08 ; p=0.007)$. In the health service evaluation block, the association found was the belief that the guidance helped to learn about pregnancy and child care $(\mathrm{OR}=1.24$; $\mathrm{CI} 95 \%=1.05-1.46 ; p=0.012)$, evaluating the $\mathrm{BHU}$ as very good/good $(\mathrm{OR}=1.19 ; \mathrm{CI}=95 \%=1.14-1.24$; $p<0.001)$, and did not want to change the BHU or the health team $(\mathrm{OR}=1.62 ; \mathrm{CI} 95 \%=1.48-1.68 ; p<0.001)$.

Table 2

Prenatal factors associated to the self-perceived evaluation of care received from the health team by female users of the Primary Health Care. Brazil, 2014.

\begin{tabular}{|c|c|c|c|c|c|c|c|}
\hline \multirow[t]{3}{*}{ Variables } & \multicolumn{6}{|c|}{ Self-perceived evaluation of care received from the health team } & \multirow[t]{3}{*}{$\boldsymbol{p}^{*}$} \\
\hline & \multicolumn{2}{|c|}{ Total } & \multicolumn{2}{|c|}{$\begin{array}{l}\text { Very good/ } \\
\text { Good }\end{array}$} & \multicolumn{2}{|c|}{$\begin{array}{l}\text { Regular / Bad / } \\
\text { Very bad }\end{array}$} & \\
\hline & $\mathrm{n}$ & $\%$ & $\mathrm{n}$ & $\%$ & $\mathrm{n}$ & $\%$ & \\
\hline \multicolumn{8}{|l|}{ Clinical and Laboratory procedures } \\
\hline Did you have your belly measured $?(n=9649)$ & & & & & & & $<0.001$ \\
\hline No & 315 & 3.3 & 214 & 2.7 & 101 & 5.7 & \\
\hline Yes & 9334 & 96.7 & 7668 & 97.3 & 1666 & 94.3 & \\
\hline \multicolumn{3}{|l|}{ Did you have your blood pressure checked? $(n=9922)$} & & & & & $<0.001$ \\
\hline No & 115 & 1.2 & 75 & 0.9 & 40 & 2.2 & \\
\hline Yes & 9807 & 98.8 & 8040 & 99.1 & 1767 & 97.8 & \\
\hline \multicolumn{2}{|l|}{ Did you have your mouth examined? $(n=9922)$} & & & & & & $<0.001$ \\
\hline No & 5038 & 50.8 & 3776 & 46.5 & 1262 & 69.8 & \\
\hline Yes & 4884 & 49.2 & 4339 & 53.5 & 545 & 30.2 & \\
\hline \multicolumn{2}{|l|}{ Did you have your breasts examined? $(n=9922)$} & & & & & & $<0.001$ \\
\hline No & 4334 & 43.7 & 3204 & 39.5 & 1130 & 62.5 & \\
\hline Yes & 5588 & 56.3 & 4911 & 60.5 & 677 & 37.5 & \\
\hline \multicolumn{2}{|l|}{ Did you have a Papanicolaou test? $(n=9922)$} & & & & & & $<0.001$ \\
\hline No & 6422 & 64.7 & 5125 & 63.2 & 1297 & 71.8 & \\
\hline Yes & 3500 & 35,3 & 2990 & 36.8 & 510 & 28.2 & \\
\hline \multicolumn{2}{|l|}{ Did you get a gynecological examination? $(n=9922)$} & & & & & & $<0.001$ \\
\hline No & 5811 & 58.6 & 4566 & 56.3 & 1245 & 68.9 & \\
\hline Yes & 4111 & 41.4 & 3549 & 43.7 & 562 & 31.1 & \\
\hline
\end{tabular}

SAH= systemic arterial hypertension; HIV= human immunodeficiency virus; VDLR= Venereal Disease Research Laboratory examination; ${ }^{\star}$ Chi-square test $(p<0.05)$.

continue 
Prenatal factors associated to the self-perceived evaluation of care received from the health team by female users of the Primary Health Care. Brazil, 2014.

\begin{tabular}{|c|c|c|c|c|c|c|c|}
\hline \multirow[t]{3}{*}{ Variables } & \multicolumn{6}{|c|}{ Self-perceived evaluation of care received from the health team } & \multirow[t]{3}{*}{$p^{*}$} \\
\hline & \multicolumn{2}{|c|}{ Total } & \multicolumn{2}{|c|}{$\begin{array}{l}\text { Very good/ } \\
\text { Good }\end{array}$} & \multicolumn{2}{|c|}{$\begin{array}{l}\text { Regular / Bad / } \\
\text { Very bad }\end{array}$} & \\
\hline & $\mathrm{n}$ & $\%$ & $\mathrm{n}$ & $\%$ & $\mathrm{n}$ & $\%$ & \\
\hline \multicolumn{8}{|l|}{ Clinical and Laboratory procedures } \\
\hline Did you have a urine test? $(n=9846)$ & & & & & & & $<0.001$ \\
\hline No & 239 & 2.4 & 175 & 2.2 & 64 & 3.6 & \\
\hline Yes & 9607 & 97.6 & 7883 & 97.8 & 1724 & 96.4 & \\
\hline Did you get an HIV examination? $(n=9726)$ & & & & & & & $<0.001$ \\
\hline No & 504 & 5.2 & 369 & 4.6 & 135 & 7.7 & \\
\hline Yes & 9922 & 94.8 & 7593 & 95.4 & 1629 & 92.3 & \\
\hline Did you get the VDRL examination? $(n=8835)$ & & & & & & & $<0.001$ \\
\hline No & 1116 & 12.6 & 800 & 11.0 & 316 & 19.9 & \\
\hline Yes & 7769 & 87.4 & 6497 & 89.0 & 1272 & 80.1 & \\
\hline Did you get an ultrasound examination? $(n=9736)$ & & & & & & & $<0.001$ \\
\hline No & 592 & 6.1 & 157 & 8.9 & 435 & 5.5 & \\
\hline Yes & 9144 & 93.9 & 7540 & 91.1 & 1604 & 94.5 & \\
\hline Did you have a glucose test? $(n=9570)$ & & & & & & & $<0.001$ \\
\hline No & 1338 & 14.0 & 939 & 12.0 & 399 & 23.2 & \\
\hline Yes & 8232 & 86.0 & 6909 & 88.0 & 1323 & 76.8 & \\
\hline Did you get a tetanus vaccination? $(n=9692)$ & & & & & & & $<0.001$ \\
\hline No & 1048 & 10.8 & 802 & 10.1 & 246 & 14.0 & \\
\hline Yes & 8644 & 89.2 & 7135 & 89.9 & 1509 & 86.0 & \\
\hline Did you get a ferrous sulfate prescription? $(n=9874)$ & & & & & & & $<0.001$ \\
\hline No & 326 & 3.3 & 249 & 3.1 & 77 & 4.3 & \\
\hline Yes & 9548 & 96.7 & 7829 & 96.9 & 1719 & 95.7 & \\
\hline Were you prescribed vitamin B9? $(n=9814)$ & & & & & & & $<0.001$ \\
\hline No & 659 & 6.7 & 492 & 6.1 & 167 & 9.4 & \\
\hline Yes & 9155 & 93.3 & 7538 & 93.9 & 1617 & 90.6 & \\
\hline Number of appointments? $(n=9284)$ & & & & & & & $<0.001$ \\
\hline Up to 5 & 1461 & 15.7 & 1137 & 14.9 & 324 & 19.3 & \\
\hline 6 appointments or over & 7823 & 84.3 & 6470 & 85.1 & 1353 & 80.7 & \\
\hline \multicolumn{8}{|l|}{ Did you need emergency care during } \\
\hline pregnancy? $(\mathrm{n}=9893)$ & & & & & & & $<0.001$ \\
\hline No & 6616 & 66.9 & 5476 & 67.6 & 1140 & 63.4 & \\
\hline Yes & 3277 & 33.1 & 2619 & 32.4 & 658 & 36.6 & \\
\hline \multicolumn{8}{|l|}{ Service organization } \\
\hline Was prenatal care provided by a physician? $(n=9136)$ & & & & & & & $<0.001$ \\
\hline No & 2333 & 25.5 & 1793 & 24.1 & 540 & 32.1 & \\
\hline Yes & 6806 & 74.5 & 5662 & 75.9 & 1141 & 67.9 & \\
\hline Was prenatal care provided by a nurse? $(n=9136)$ & & & & & & & $<0.001$ \\
\hline No & 2400 & 26.3 & 2026 & 27.2 & 374 & 22.2 & \\
\hline Yes & 6736 & 73.7 & 5429 & 72.8 & 1307 & 77.8 & \\
\hline
\end{tabular}

$\mathrm{SAH}=$ systemic arterial hypertension; HIV= human immunodeficiency virus; VDLR= Venereal Disease Research Laboratory examination; ${ }^{*}$ Chi-square test $(p<0.05)$. 
Prenatal factors associated to the self-perceived evaluation of care received from the health team by female users of the Primary Health Care. Brazil, 2014.

\begin{tabular}{|c|c|c|c|c|c|c|c|}
\hline \multirow[t]{3}{*}{ Variables } & \multicolumn{6}{|c|}{ Self-perceived evaluation of care received from the health team } & \multirow[t]{3}{*}{$p^{*}$} \\
\hline & \multicolumn{2}{|c|}{ Total } & \multicolumn{2}{|c|}{$\begin{array}{l}\text { Very good/ } \\
\text { Good }\end{array}$} & \multicolumn{2}{|c|}{$\begin{array}{l}\text { Regular / Bad / } \\
\text { Very bad }\end{array}$} & \\
\hline & $\mathrm{n}$ & $\%$ & $\mathrm{n}$ & $\%$ & $\mathrm{n}$ & $\%$ & \\
\hline \multicolumn{8}{|l|}{ Service organization } \\
\hline \multicolumn{8}{|l|}{ Did you have appointments with the same } \\
\hline professional? $(\mathrm{n}=9922)$ & & & & & & & $<0.001$ \\
\hline No & 1323 & 13.3 & 940 & 11.6 & 383 & 21.1 & \\
\hline Yes & 8599 & 86.7 & 7171 & 88.4 & 1428 & 78.9 & \\
\hline \multicolumn{8}{|c|}{ Did you leave the BHU with the next appointment } \\
\hline already scheduled? $(n=9892)$ & & & & & & & $<0.001$ \\
\hline Never / Almost never/ Usually & 1323 & 13.4 & 940 & 11.6 & 383 & 21.3 & \\
\hline Always & 8569 & 86.6 & 7151 & 88.4 & 1418 & 78.7 & \\
\hline \multicolumn{8}{|l|}{ Health guidance } \\
\hline Instructions about diet/weight ( $n=9922)$ & & & & & & & $<0.001$ \\
\hline No & 1194 & 12.0 & 742 & 9.1 & 452 & 25.0 & \\
\hline Yes & 8728 & 88.0 & 9393 & 90.1 & 1355 & 75.0 & \\
\hline Instructions about breastfeeding ( $\mathrm{n}=9922)$ & & & & & & & $<0.001$ \\
\hline No & 915 & 9.2 & 560 & 7.0 & 351 & 19.4 & \\
\hline Yes & 9007 & 90.8 & 7551 & 93.0 & 1456 & 80.6 & \\
\hline Instructions about childcare ( $\mathrm{n}=9922)$ & & & & & & & $<0.001$ \\
\hline No & 1467 & 14.8 & 920 & 11.3 & 547 & 30.3 & \\
\hline Yes & 8455 & 85.2 & 7195 & 88.7 & 1260 & 69.7 & \\
\hline \multicolumn{8}{|l|}{ Instructions about the gynecological preventive } \\
\hline exam $(n=9922)$ & & & & & & & $<0.001$ \\
\hline No & 3443 & 34.7 & 2450 & 30.2 & 993 & 55.0 & \\
\hline Yes & 6479 & 65.3 & 5665 & 69.8 & 814 & 45.0 & \\
\hline \multicolumn{8}{|c|}{ Instructions about the pregnant woman support } \\
\hline group $(n=9816)$ & & & & & & & $<0.001$ \\
\hline No & 4432 & 45.2 & 3332 & 41.5 & 1100 & 61.7 & \\
\hline Yes & 5384 & 54.8 & 4700 & 58.5 & 684 & 38.3 & \\
\hline \multicolumn{8}{|l|}{ Participating in the pregnant woman support } \\
\hline group $(n=9838)$ & & & & & & & $<0.001$ \\
\hline No & 3859 & 39.2 & 4656 & 57.9 & 1323 & 73.9 & \\
\hline Yes & 5979 & 60.8 & 3391 & 42.1 & 468 & 26.1 & \\
\hline \multicolumn{8}{|l|}{ Getting information about the maternity } \\
\hline hospital $(n=9840)$ & & & & & & & $<0.001$ \\
\hline No & 4191 & 42.6 & 3172 & 39.4 & 1019 & 56.6 & \\
\hline Yes & 5649 & 57.4 & 4869 & 60.6 & 780 & 43.4 & \\
\hline
\end{tabular}

$\mathrm{SAH}=$ systemic arterial hypertension; HIV= human immunodeficiency virus; VDLR= Venereal Disease Research Laboratory examination; *Chi-square test $(p<0.05)$. 
Table 3

Factors in the evaluation of health services associated to the auto-perceived evaluation of care received from the health team by female users of the Primary Health Care. Brazil, 2014.

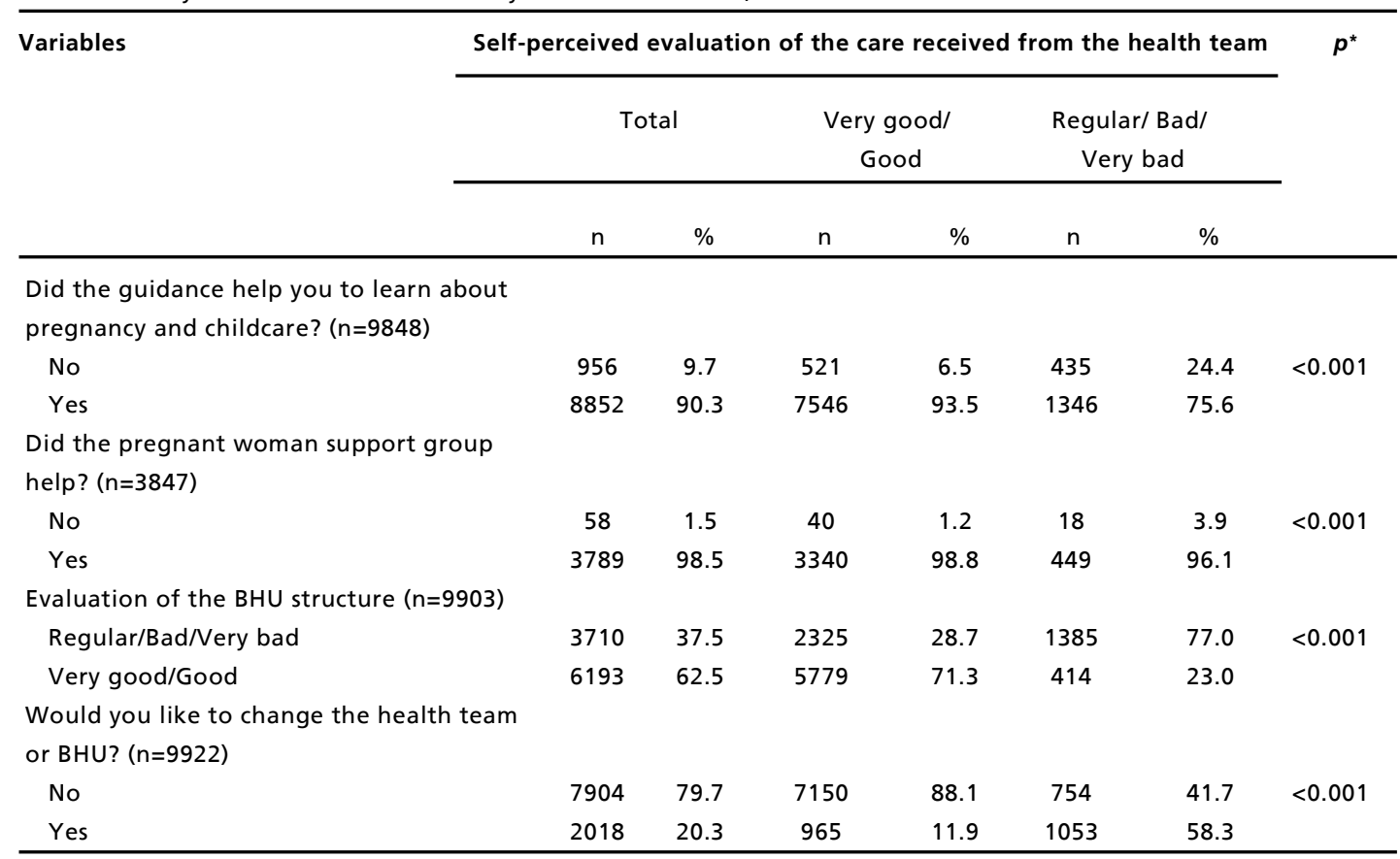

BHU $=$ Basic Health Unit; ${ }^{*}$ Chi-square test $(p<0.05)$.

\section{Table 4}

Adjusted gross analysis between the self-perceived evaluation of care received from the health team and sociodemographic factors, prenatal care and service evaluation by female users of the Primary Health Care. Brazil, 2014.

\begin{tabular}{|c|c|c|c|c|c|c|c|c|c|}
\hline \multirow[t]{3}{*}{ Variables (Ref.) } & \multicolumn{4}{|c|}{ Gross analysis } & \multicolumn{5}{|c|}{ Adjusted analysis } \\
\hline & \multirow[b]{2}{*}{ OR } & \multirow[b]{2}{*}{$\mathrm{Cl} 195 \%$} & \multirow[b]{2}{*}{$p$} & \multirow[b]{2}{*}{ OR } & \multicolumn{2}{|l|}{ Model 1} & \multicolumn{2}{|c|}{ Model 2* } & \multirow[b]{2}{*}{$p$} \\
\hline & & & & & $\mathrm{Cl} 95 \%$ & $p$ & OR & $\mathrm{Cl} 195 \%$ & \\
\hline \multicolumn{10}{|c|}{ Sociodemographic characteristics } \\
\hline \multicolumn{10}{|l|}{ Age (<20 years) } \\
\hline 20-34 years & 1.62 & $1.35-1.94$ & $<0.001$ & & & & & & \\
\hline$>34$ years & 1.97 & $1.58-2.45$ & $<0.001$ & & & & & & \\
\hline \multicolumn{10}{|l|}{ Race (Others) } \\
\hline Caucasian & 1.34 & $1.19-1.51$ & $<0.001$ & & & & & & \\
\hline \multicolumn{10}{|l|}{ Schooling ( incomplete EE) } \\
\hline Incomplete HS & 1.54 & $1.20-1.99$ & $<0.001$ & 1.00 & $0.96-1.03$ & 0.765 & 0.99 & $0.96-1.02$ & 0.462 \\
\hline Completed HS & 1.96 & $1.53-2.52$ & $<0.001$ & 1.03 & $0.99-1.07$ & 0.073 & 1.01 & $0.98-1.04$ & 0.423 \\
\hline Incomplete HE or over & 1.82 & $1.31-2.35$ & 0.001 & 1.07 & $1.04-1.13$ & $<0.001$ & 1.05 & $1.01-1.09$ & 0.010 \\
\hline \multicolumn{10}{|l|}{ Job (No) } \\
\hline Yes & 1.51 & $1.19-1.92$ & 0.001 & & & & & & \\
\hline \multicolumn{10}{|c|}{ Own income ( $<1$ minimum wage) } \\
\hline 1 or over & 1.04 & $1.02-1.06$ & $<0.001$ & & & & & & \\
\hline \multicolumn{10}{|c|}{ Bolsa Família (Not beneficiary) } \\
\hline Beneficiary & 1.04 & $1.02-1.05$ & $<0.001$ & & & & & & \\
\hline Prenatal care & & & & & & & & & continue \\
\hline
\end{tabular}

*Good-of-fit: Likelihood Ratio Chi-square Pearson: $99.344 \quad(p<0.001)$ 
Adjusted gross analysis between the self-perceived evaluation of care received from the health team and sociodemographic factors, prenatal care and service evaluation by female users of the Primary Health Care. Brazil, 2014.

\begin{tabular}{|c|c|c|c|c|c|c|c|c|c|}
\hline \multirow[t]{3}{*}{ Variables (Ref.) } & \multicolumn{3}{|c|}{ Gross analysis } & \multicolumn{6}{|c|}{ Adjusted analysis } \\
\hline & \multirow[b]{2}{*}{ OR } & \multirow[b]{2}{*}{$\mathrm{Cl} 195 \%$} & \multirow[b]{2}{*}{$p$} & \multicolumn{2}{|r|}{ Model 1} & \multicolumn{4}{|c|}{ Model $2^{*}$} \\
\hline & & & & OR & Cl95\% & $p$ & OR & $\mathrm{Cl} 95 \%$ & $p$ \\
\hline \multicolumn{10}{|c|}{ Clinical and laboratory procedures } \\
\hline \multicolumn{10}{|l|}{ Did you have your belly } \\
\hline \multicolumn{10}{|l|}{ measured? (No) } \\
\hline Yes & 2.17 & $1.70-2.77$ & $<0.001$ & & & & & & \\
\hline \multicolumn{10}{|c|}{ Did you have your blood pressure } \\
\hline \multicolumn{10}{|c|}{ checked? (No) } \\
\hline Yes & 2.43 & $1.65-3.57$ & $<0.001$ & & & & & & \\
\hline \multicolumn{10}{|l|}{ Did you have your mouth } \\
\hline \multicolumn{10}{|l|}{ examined? (No) } \\
\hline Yes & 2.66 & $2.38-2.97$ & $<0.001$ & 1.03 & $0.99-1.06$ & 0.105 & & & \\
\hline \multicolumn{10}{|l|}{ Did you have your breasts } \\
\hline \multicolumn{10}{|l|}{ examined? (No) } \\
\hline Yes & 2.56 & $2.30-2.84$ & $<0.001$ & 1.04 & $1.00-1.07$ & 0.050 & & & \\
\hline \multicolumn{10}{|l|}{ Did you get a Papanicolaou } \\
\hline \multicolumn{10}{|l|}{ test? (No) } \\
\hline Yes & 1.48 & $1.32-1.66$ & & & & & & & \\
\hline \multicolumn{10}{|l|}{ Did you get a gynecological } \\
\hline examination? (No) & & & & & & & & & \\
\hline Yes & 1.72 & $1.54-1.92$ & $<0.001$ & 0.98 & $0.95-1.00$ & 0.090 & & & \\
\hline Did you get a urine test? ( & & & & & & & & & \\
\hline Yes & 1.67 & $1.25-2.24$ & $<0.001$ & & & & & & \\
\hline Did you have an HIV & & & & & & & & & \\
\hline examination? (No) & & & & & & & & & \\
\hline Yes & 1.70 & $1.39-2.09$ & $<0.001$ & 1.12 & $1.04-1.22$ & 0.005 & & & \\
\hline Did you have a VDRL & & & & & & & & & \\
\hline examination? (No) & & & & & & & & & \\
\hline Yes & 2.02 & $1.75-2.33$ & $<0.001$ & 1.12 & $1.04-1.20$ & 0.003 & 1.07 & $1.01-1.14$ & 0.020 \\
\hline Did you have an ultrasounc & & & & & & & & & \\
\hline examination? (No) & & & & & & & & & \\
\hline Yes & 1.70 & $1.40-2.05$ & $<0.001$ & 1.07 & $0.98-1.16$ & 0.122 & & & \\
\hline Did you get a glucose test? & & & & & & & & & \\
\hline Yes & 2.22 & $1.95-2.53$ & $<0.001$ & 1.10 & $1.02-1.18$ & 0.007 & & & \\
\hline Did you get a tetanus & & & & & & & & & \\
\hline vaccination? (No) & & & & & & & & & \\
\hline Yes & 1.45 & $1.24-1.69$ & $<0.001$ & 0.90 & $0.93-1.02$ & 0.188 & & & \\
\hline Were you prescribed ferrou & & & & & & & & & \\
\hline sulfate? (No) & & & & & & & & & \\
\hline Yes & 1.41 & $1.08-1.83$ & $<0.001$ & & & & & & \\
\hline Were you prescribed vitam & & & & & & & & & \\
\hline B9? (No) & & & & & & & & & \\
\hline Yes & 1.58 & $1.32-1.90$ & $<0.001$ & & & & & & \\
\hline Number of appointments & & & & & & & & & \\
\hline 6 appointments or over & 1.08 & $1.04-1.12$ & $<0.001$ & 0.96 & $0.92-1.02$ & 0.162 & & & \\
\hline Did you need emergency ce & & & & & & & & & \\
\hline during pregnancy (yes) & & & & & & & & & \\
\hline No & 1.03 & $1.00-1.05$ & $<0.001$ & & & & & & \\
\hline
\end{tabular}

*Good-of-fit: Likelihood Ratio Chi-square Pearson: $99.344 \quad(p<0.001)$. 


\section{Table 4}

Adjusted gross analysis between the self-perceived evaluation of care received from the health team and sociodemographic factors, prenatal care and service evaluation by female users of the Primary Health Care. Brazil, 2014.

\begin{tabular}{|c|c|c|c|c|c|c|c|c|c|}
\hline \multirow[t]{3}{*}{ Variables (Ref.) } & \multicolumn{3}{|c|}{ Gross analysis } & \multicolumn{6}{|c|}{ Adjusted analysis } \\
\hline & & & & \multicolumn{3}{|c|}{ Model 1} & \multicolumn{3}{|c|}{ Model 2* } \\
\hline & OR & C195\% & $p$ & OR & $\mathrm{Cl} 195 \%$ & $p$ & OR & $\mathrm{Cl} 95 \%$ & $p$ \\
\hline
\end{tabular}

Service organization

Was the prenatal care provided

by a physician? (No)

Yes

$1.50 \quad 1.33-1.68<0.001$

Was the prenatal care provided by a nurse? (Yes)

Were all your appointments with

the same professional? (No)

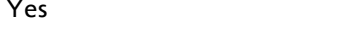

$\begin{array}{llllll}1.03 & 1.01-1.05 & 0.011 & 1.04 & 1.01-1.07 & 0.017\end{array}$

Did you leave the BHU with the

next appointment already scheduled?

(Never/ Almost never / usually)

$\begin{array}{lllllll}\text { Always } & 2.06 & 1.80-2.34 & <0.001 & 1.09 & 1.03-1.16 & 0.002\end{array}$

Health guidance

Instructions about diet/weight (No)

Yes

$\begin{array}{llllll}3.31 & 2.91-3.78 & <0.001 & 1.08 & 0.98-1.19 & 0.133\end{array}$

Instructions about

breastfeeding (No)

Yes

$3.23 \quad 2.79-3.73<0.001$

Instructions about child care (No)

Yes

$\begin{array}{llllll}3.40 & 3.01-3.83 & <0.001 & 1.14 & 1.03-1.26 & 0.008\end{array}$

Instructions about gynecological

preventive examination (No)

Yes

$\begin{array}{lllllllll}2.82 & 2.54-3.13 & <0.001 & 1.07 & 1.02-1.11 & 0.001 & 1.05 & 1.01-1.08 & 0.007\end{array}$

Instructions about pregnant

woman support group (No)

Yes

$2.27 \quad 2.04-2.52<0.001$

Participating in the pregnant

woman support group (No)

Yes

$2.061 .84-2.31<0.001$

Information about the

maternity hospital (No)

Yes

$\begin{array}{llllll}2.00 & 1.81-2.22 & <0.001 & 1.03 & 0.99-1.06 & 0.061\end{array}$

*Good-of-fit: Likelihood Ratio Chi-square Pearson: $99.344 \quad(p<0.001)$ 
Adjusted gross analysis between the self-perceived evaluation of care received from the health team and sociodemographic factors, prenatal care and service evaluation by female users of the Primary Health Care. Brazil, 2014.

\begin{tabular}{|c|c|c|c|c|c|c|c|c|c|}
\hline \multirow[t]{3}{*}{ Variables (Ref.) } & \multicolumn{3}{|c|}{ Gross analysis } & \multicolumn{6}{|c|}{ Adjusted analysis } \\
\hline & \multirow[b]{2}{*}{ OR } & \multirow[b]{2}{*}{$\mathrm{Cl} 95 \%$} & \multirow[b]{2}{*}{$p$} & \multicolumn{2}{|c|}{ Model 1} & & \multicolumn{2}{|c|}{ Model 2* } & \multirow[b]{2}{*}{$p$} \\
\hline & & & & OR & $\mathrm{Cl} 95 \%$ & $p$ & OR & $\mathrm{Cl} 95 \%$ & \\
\hline \multicolumn{10}{|l|}{ Service evaluation } \\
\hline \multicolumn{9}{|c|}{ Did guidance help to learn about } & \\
\hline Yes & 4.68 & $4.07-5.38$ & $<0.001$ & & & & 1.24 & $1.05-1.46$ & 0.012 \\
\hline \multicolumn{9}{|c|}{ Pregnant woman group } & helped (No) \\
\hline Yes & 3.35 & $1.90-5.89$ & $<0.001$ & & & & & & \\
\hline \multicolumn{9}{|c|}{ BHU structure evaluation } & (Regular/Bad/Very bad) \\
\hline Very good/Good & 8.32 & 7.37-9.37 & $<0.001$ & & & & 1.19 & $1.14-1.24$ & $<0.001$ \\
\hline \multicolumn{9}{|c|}{ Desire to change team or } & \\
\hline No & 1.86 & 1.77-1.95 & $<0.001$ & & & & 1.62 & $1.48-1.78$ & $<0.001$ \\
\hline
\end{tabular}

*Good-of-fit: Likelihood Ratio Chi-square Pearson: $99.344 \quad(p<0.001)$.

\section{Discussion}

In this study, most women who received prenatal care in the Basic Health Unit investigated evaluated positively the care provided by the health team regarding primary health care as very good/good. The literature still lacks studies evaluating prenatal care from the women's standpoint, that is, considering the users' opinion or satisfaction in relation to the prenatal care received.14-21 The studies found seek to evaluate the quality of services through parameters and/or indicators such as number of appointments, early start of assistance, examination carried out and medication prescription, ${ }^{8-13}$ hampering possible comparisons in this scenery with the findings of this study.

Although studies measuring health indicators in the perspective of the installed capacity or the service offered point out low quality in the public health service prenatal care, $8-13$ studies considering the users' perspective have reported positive perception, ${ }^{15-21}$ as in this study. Studies carried out in Brazil and abroad, in Belgium 20 and Colombia, 21 showed that although the participant women initially tended to have low expectancy, in general they reported satisfaction with the prenatal care received. This discrepancy reveals that the evaluation that only considers quantitative aspects of the clinical indicators disregards an important factor, the users' satisfaction. For example, a pregnant woman might not have had the minimum number of appointments, since she only discovered her pregnancy later, or for personal reasons, and therefore, she did not have all the laboratory exams, however, from the moment she accessed the service onwards, she received good care from the health team regarding childcare guidance. While this woman would have a bad prenatal care in an evaluation of the quantitative indicators only, the same patient could reveal a good selfperceived evaluation of her prenatal care, since from the moment she contacted the health service onwards, she was well assisted and guided by the health team.

The best self-perceived evaluation regarding prenatal care was that associated to the women's higher schooling, an aspect also found in objective measurement studies, in which the higher the mother's schooling was, the lower the chances of an unsuitable prenatal were found.15,23-25 In addition, low schooling might interfere in the perception of an individual's health or of health services. 26 The hypothesis in this case is that the higher the schooling is, the better the understanding of the importance of the prenatal care is, or even, whether there is inequality in the assistance provided by the professionals to more educated women, since they would understand more easily the information received and/or really receive more attention from 
the team. Therefore, the professionals should reflect upon the need for a more inclusive professional practice with the purpose of qualifying all mothers to have a minimum suitable care and a safe prenatal period, by adopting shared responsibility.

Within the prenatal care, when the clinical and laboratory parameters were evaluated, the best service quality was associated to those that had the VDRL examination. Carrying out laboratory examination is an essential measure to prevent, identify, minimize or solve problems related to the mother's or fetus' health during pregnancy. The lack of such examinations is directly related to high rates of syphilis vertical transmission and occurrence of perinatal deaths, thus pointing out some of the problems in the service rendered.8,24 Therefore, the hypothesis arises that pregnant women who had fewer examinations might have had more complications and possible fetal malformation, and for this reason they evaluated the prenatal care as bad. Conversely, those that had a higher number of examinations had a favorable outcome and thus a more positive perception. Although several studies reporting objective evaluation of the prenatal care showed that the more laboratory and clinical examinations were carried out, the better the suitability of the assistance was, 10,27 this study verified that other factors were far more relevant in the users' self-perceived evaluation of the care received.

As for the service organization, the women that had the prenatal care provided by the same professional evaluated better the service received. Although comparative data was not found, a study carried out at the Primary Health Care - PHC (Atenção Primária à Saúde - APS) in Bauru-SP, verified that the users' prenatal care satisfaction was associated to the prenatal care being received in the same health unit. 18 This result is due to the longitudinal care, an essential feature of the PHC, which provides continuity to the assistance and a closer relationship between the health professionals and the users. 28 A study carried out in Colombia showed that the interaction with the professional that renders the service was considered a determining factor of the users' level of satisfaction with the public service and of the adhesion to the prenatal care service. ${ }^{21}$

When health guidance was analyzed, receiving guidance on the preventive gynecological examination was associated to better self-perceived evaluation of the care received by the users, as evidenced in other studies. 12,13 In addition, in this study, the mothers that stated that this guidance helped them to learn about pregnancy and childcare presented a more positive perception of the service received.
These findings might result from the humanization of the services, greater safety due to the instructions given by the health team and the current health policies regarding the use of soft technologies in health care. 29

Regarding the importance of the use of soft technologies, a study developed in Turkey showed that pregnant women that received health education in the first prenatal appointment presented fewer complications during pregnancy. 30 This data evidences the importance of health education for a positive perception of the prenatal care. For this reason, professionals and scholars in the nursing and medical area should emphasize the topics related to health education and provide suitable guidance to the pregnant women in prenatal appointments, since this is a cheap resource that has a positive result in quality indices.

The best evaluation of the BHU facilities was associated to a better self-perceived evaluation of the care received from the team. This aspect might be associated to the atmosphere issue, an aspect also considered in the National Humanization Policy. 29 When arriving at a place that provides health assistance, a condition of salubrity and care consistent with the hygiene aspect is expected. The absence of such an environment might affect negatively the service rendered. Therefore, it seems relevant to emphasize that despite all work developed to incorporate a structural improvement to the BHU through financial support such as the PMAQ-AB, there are still limitations that need to be spotted and addressed by the municipal management teams in the BHU infrastructure.

The participants that best evaluated the service received showed the least intention of changing the BHU or the health team. Studies developed in Brazil and Colombia, both developing countries, showed that satisfied users tend to recommend the service to other people.18,21 This aspect might be associated to the perception of safety, therefore, they want to continue receiving this care at the $\mathrm{BHU}$ or from the same team, and also want that their relations benefit from it.

This study showed that the best users' evaluation was associated to the continuous care and the quality of health guidance received, rather than the laboratory and/or clinical procedures carried out. Therefore, the perception of care found was more associated to soft technologies that surpassed the hard ones. 30 For this reason, policies such as the PMAQ-AB, which evaluate and favor quality not only quantity, become more and more important in the health care environment. 
One limitation of this study might be the use of secondary data due to problems related to the quality and accuracy of the data, such as for example, information bias. Since data from the PMAQ-AB was used, we assumed that only teams that volunteered took part in the evaluation process. Therefore, for not being a sample of all Primary Health Care Team in Brazil, it might not represent the reality throughout the country. There is also the possibility that the values do not express the reality of the entire Brazilian population, since the interviewees had access to the PHC, which is not the reality of the more disadvantage population in our country. Another limitation could be the memory bias due to the time of the interview, since the women were not interviewed while they were pregnant, but only after the gestational period.

The main strength of the study is the valorization of the users' evaluation of the prenatal care received, inserting them as part of the evaluation process, giving a more humanized character to the prenatal care. In addition, the use of great amount of information and data can be considered a strength, since it was possible to evaluate the self-perceived quality of the prenatal care at a national level. Therefore, the PMAQ-AB was seen to be a useful tool to evaluate a large number of items related to the PHC in Brazil, 7 including the prenatal care provided by the Primary Health care.

\section{References}

1. Rio Grande do Sul. Secretaria de Estado da Saúde. Guia do pré-natal na atenção básica; 2018.

2. Barbeiro FMS, Fonseca SC, Tauffer MG, Ferreira MSS Silva FP, Ventura PV, et al. Óbitos fetais no Brasil: revisão sistemática. Rev Saúde Pública. 2015; 49: 22.

3. Pereira DO, Ferreira TLS, Araújo DV, Melo KDF, Andrade FB. Avaliação das consultas de pré-natal: adesão do prénatal e complicações na saúde materno-infantil. Rev Ciência Plural. 2017; 3 (3): 2-15.

4. Justino DCP, Lopes MS, Santo CDP, Andrade FB Avaliação histórica das políticas públicas de saúde infantil no brasil: revisão integrativa. Rev Ciênc Plural. 2019; 5 (1) 71-88.

5. WHO (World Health Organization). Millennium Declaration. Declaration of the United Nations General Assembly; 2000.

6. Santos HFL, Araujo MM. Políticas de humanização ao prénatal e parto: uma revisão de literatura. Rev Cient FacMais 2016; 6 (2): 55-64

7. Pinto HA, Sousa ANA, Ferla AA. O Programa Nacional de Melhoria do Acesso e da Qualidade da Atenção Básica várias faces de uma política inovadora. Saúde Debate.
Our results led to the conclusion that the selfperceived positive evaluation of the care received from the health team was associated to sociodemographic factors, prenatal assistance and health service evaluation among the women that had their prenatal service provided by the Primary Health Care in Brazil.

The users' perspective must be increasingly taken into consideration by health managers and professionals. In this regard, managers cannot stop to seek for improvement of the quantitative indicators related to the prenatal care such as clinical and/or laboratory procedures and at the same time promote a humanized care that includes the users' views.

This study revealed that in the users' perspective, factors associated to soft technologies such as the longitudinal bond with the team and health guidance were factors strongly associated to the positive evaluation given to the service rendered by the health team.

\section{Author's contribution}

Di Dea B and Andrade F organized the database, prepared the table and wrote the scientific article; Silva-Junior MF participated in the study design, data analysis and final review of the article. All authors approved the final version of the article.
2014; 38 (esp.): 358-72.

8. Balsells MMD, Oliveira TMF, Bernardo EBR, Aquino PS, Damasceno AKC, Castro RCMB, Lessa PRA, Pinheiro AKB. Avaliação do processo na assistência pré-natal de gestantes com risco habitual. Acta Paulista Enferm. 2018; 31 (3): 247-54.

9. Nunes JT, Gomes KRO, Rodrigues MTP, Mascarenhas MDM. Qualidade da assistência pré-natal no Brasil: revisão de artigos publicados de 2005 a 2015. Cad Saúde Coletiva. 2016; 24 (2): 252-61.

10. Paris GF, Pelloso SM, Martins PM. Qualidade da assistência pré-natal nos serviços públicos e privados. Rev Bras Ginecol Obstet. 2013; 35 (10): 447-52.

11. Viellas EF, Domingues RMSM, Dias MAB, Gama SGN, Filha MMT, Costa JV. Prenatal care in Brazil. Cad Saúde Pública. 2014; 30: S85-S100.

12. Tomasi E, Fernandes PAA, Fischer T, Siqueira SCV, Silveira DS, Thumé E, Duro SMS, Saes MO, Nunes BP, Fassa AG, Facchini L. Qualidade da atenção pré-natal na rede básica de saúde do Brasil: indicadores e desigualdades sociais. Cad Saúde Pública. 2017; 33 (3): e00195815.

13. Domingues RMSM, Hartz ZMA, Dias MAB, Leal MC. 
Avaliação da adequação da assistência pré-natal na rede SUS do Município do Rio de Janeiro, Brasil. Cad Saúde Pública. 2012; 28 (3): 425-37.

14. Silveira LI, Andrade F, Dea BD, Muller EV, Silva Junior MF. Fatores associados ao número de consultas no prénatal: análise segundo a autopercepção de usuárias da atenção primária no Brasil. Arq Catarin Med. 2020; 49 (2): 29-42.

15. Domingues RMSM, Viellas EF, Dias MAB, Torres JA Theme-Filha MM, Gama SGN, Leal MC. Adequação da assistência pré-natal segundo as características maternas no Brasil. Rev Panam Salud Pública. 2015; 37 (3): 140-7.

16. Silva ALA, Mendes ACG, Miranda GMD, Souza WV. A qualidade do atendimento ao parto na rede pública hospitalar em uma capital brasileira: a satisfação das gestantes. Cad Saúde Pública. 2017; 33(12): e00175116.

17. Santos AL, Radovanovic CAT, Marcon SS. Assistência prénatal: satisfação e expectativas. Rev Rene. 2010; 11: 61-71.

18. Prudêncio OS, Mamede FV. Avaliação do cuidado pré-natal na atenção primária a saúde na percepção da gestante. Rev Gaúcha de Enferm. 2018; 39: e20180077.

19. Cardelli AAM, Marrero TL, Ferrari RAP, Martins JT, Serafim D. Expectations and satisfaction of pregnant women: unveiling prenatal care in primary care. Invest Educ Enferm. 2016; 34 (2): 252-60.

20. Galle A, Parys ASV, Roelens K, Keygnaet I. Expectations and satisfaction with antenatal care among pregnant women with a focus on vulnerable groups: a descriptive study in Ghent. BMC Womens Health. 2015; 15: 112.

21. Ávilla IYC, Villanueva MPV, Correa EO, Consuegra AP, Soto HC. Satisfacción de usuarias del control prenatal en instituciones de salud públicas y factores asociados. Hacia Promoción Salud. 2014; 19(1): 128-40.

22. Lima S, Carvalho M, Vasconcelos AG. Proposta de modelo hierarquizado aplicado à investigação de fatores de risco de óbito infantil neonatal. Cad Saúde Pública. 2008; 24 (8) 1910-6

Received on August 11, 2020

Final version presented on December 14, 2020

Approved on February 17, 2021
23. Fonseca SC, Monteiro DAS, Pereira CMSM, Scoralick ACD, Jorge MG Rozario S. Desigualdades no pré-natal em cidade do Sudeste do Brasil. Ciênc Saúde Coletiva. 2014; 19 (7): 1992-8.

24. Rasia ICRB, Albernaz E. Atenção pré-natal na cidade de Pelotas, Rio Grande do Sul, Brasil. Rev Bras Saúde Mater Infant. 2008; 8 (4): 401-10.

25. Ribeiro ER, Guimarães AMD, Bettiol H, Lima DF, Almeida ML, Souza L, Silva AAM, Gurgem RQ. Risk factors for inadequate prenatal care use in the metropolitan area of Aracaju, Northeast Brazil. BMC Pregnancy Childbirth. 2009; 9 (1): 31-8.

26. Agostinho MR, Oliveira MC, Pinto MEB, Balardin GU, Harzheim E. Autopercepção da saúde entre usuários da Atenção Primária em Porto Alegre, RS. Rev Bras Med Fam Comunidade. 2010; 5 (17): 9-15.

27. Valente MMCP, Freitas NQ, Afio ACE, Souza CSP, Evangelista DR, Moura ERF. Assistência pré-natal: um olhar sobre a qualidade. Rev Rene. 2013; 14 (2): 280-9.

28. Santos ROM, Romano VF, Engstrom EM. Vínculo longitudinal na Saúde da Família: construção fundamentada no modelo de atenção, práticas interpessoais e organização dos serviços. Physis: Rev Saúde Coletiva. 2018; 28 (2): 1-18.

29. Rodrigues FR, Covos JS, Covos JF, Rodrigues BC. Prénatal humanizado: estratégias de enfermagem na preparação para o parto ativo. Rev Saúde Foco. 2018; 9 (10): 89-100.

30. Yikar K, Nazik E. Effects of prenatal education on complaints during pregnancy and on quality of life. Patient Educ Couns. 2018; 102 (1): 119-25. 